This is an electronic reprint of the original article. This reprint may differ from the original in pagination and typographic detail.

Author(s): Kimme, Lukas; Hyart, Timo

Title: $\quad$ Existence of zero-energy impurity states in different classes of topological insulators and superconductors and their relation to topological phase transitions

Year: $\quad 2016$

Version:

Please cite the original version:

Kimme, L., \& Hyart, T. (2016). Existence of zero-energy impurity states in different classes of topological insulators and superconductors and their relation to topological phase transitions. Physical Review B, 93(3), Article 035134. https://doi.org/10.1103/PhysRevB.93.035134

All material supplied via JYX is protected by copyright and other intellectual property rights, and duplication or sale of all or part of any of the repository collections is not permitted, except that material may be duplicated by you for your research use or educational purposes in electronic or print form. You must obtain permission for any other use. Electronic or print copies may not be offered, whether for sale or otherwise to anyone who is not an authorised user. 


\title{
Existence of zero-energy impurity states in different classes of topological insulators and superconductors and their relation to topological phase transitions
}

\author{
Lukas Kimme $^{1}$ and Timo Hyart ${ }^{2}$ \\ ${ }^{1}$ Institut für Theoretische Physik, Universität Leipzig, D-04103 Leipzig, Germany \\ ${ }^{2}$ University of Jyväskylä, Department of Physics and Nanoscience Center, P.O. Box 35 (YFL), FI-40014 University of Jyväskylä, Finland
}

(Received 24 October 2015; published 25 January 2016)

\begin{abstract}
We consider the effects of impurities on topological insulators and superconductors. We start by identifying the general conditions under which the eigenenergies of an arbitrary Hamiltonian $H$ belonging to one of the Altland-Zirnbauer symmetry classes undergo a robust zero energy crossing as a function of an external parameter which can be, for example, the impurity strength. We define a generalized root of det $H$ and use it to predict or rule out robust zero-energy crossings in all symmetry classes. We complement this result with an analysis based on almost degenerate perturbation theory, which allows a derivation of the asymptotic low-energy behavior of the ensemble averaged density of states $\rho \sim E^{\alpha}$ for all symmetry classes and makes it transparent that the exponent $\alpha$ does not depend on the choice of the random matrix ensemble. Finally, we show that a lattice of impurities can drive a topologically trivial system into a nontrivial phase, and in particular we demonstrate that impurity bands carrying extremely large Chern numbers can appear in different symmetry classes of two-dimensional topological insulators and superconductors. We use the generalized root of det $H(\boldsymbol{k})$ to reveal a spiderweblike momentum space structure of the energy gap closings that separate the topologically distinct phases in $p_{x}+i p_{y}$ superconductors in the presence of an impurity lattice.
\end{abstract}

DOI: 10.1103/PhysRevB.93.035134

\section{INTRODUCTION}

Ever since the concept of topological order in condensed matter systems picked up momentum during the last decade [1-9], there has been the question as to which kind of perturbations can cause topological phase transitions. In this paper, we investigate the possibility to utilize inhomogeneous perturbations, like those caused by impurity lattices, for this purpose. We point out that such perturbations are often naturally present in candidate materials for topological superconductors, since these are typically unconventional superconductors obtained by doping Mott insulators [8,10-14]. Moreover, high quality topological insulators have recently been created by intentionally doping $\mathrm{Si}$ into $\mathrm{InAs} / \mathrm{GaSb}$ heterostructures [15]. Finally, a lattice of magnetic atoms placed on top of a superconductor may be a route towards the realization of a rich variety of topological phases [16]. In principle, there is also a relation to the studies about so-called topological Anderson insulators [17,18], disorderdriven topological superconductivity $[19,20]$, and impurity bound states as a signature of a topologically nontrivial phase $[21,22]$.

To intuitively understand why impurity lattices may be of interest for the design of topologically nontrivial phases, a useful starting point is to realize that nontrivial topological invariants are associated with momentum space topological defects [23], which can be inserted into the system when the bulk energy gap closes. The impurities reduce the translational symmetry of the system and give rise to impurity bands, which can have complicated energy-momentum dispersion relations described by long-range hopping terms in the low-energy theory. This allows more freedom to deform the dispersion in such a way that many band inversions appear, giving rise to topological defects in momentum space. As a result these impurity bands can carry very large topological invariants. Recently, Röntynen and Ojanen theoretically demonstrated the possibility of topological superconductivity with very large Chern numbers in 2D ferromagnetic Shiba lattices using this kind of approach [16].

In the first part of this paper (Secs. II and III), we investigate whether the eigenstates of an arbitrary Hamiltonian $H_{\lambda}=H_{0}+\lambda H_{1}$ belonging to one of the ten Altland-Zirnbauer symmetry classes $[24,25]$ undergo robust zero-energy crossings as a function of $\lambda$. In this regard, there are two main results: (i) We define the generalized root $q(H)$ of det $H$ in such a way that $|q(H)|^{v}=|\operatorname{det} H|$, and $q(H)$ is a polynomial function of the matrix elements of the Hamiltonian. Here, $v$ is the symmetry-induced degeneracy of eigenstates, when eigenstates at $\pm E$ are considered degenerate. We show that $q(H)$ is real in classes $\mathrm{A}, \mathrm{AI}, \mathrm{AII}, \mathrm{BDI}$, and $\mathrm{D}$, it is complex in classes AIII, CI, and DIII, and it cannot be defined in classes $\mathrm{C}$ and CII. When $q$ is real, $\operatorname{sgn} q(H)$ equals the parity of the topological invariant in $d=0$ dimensions $[3,9,21,26,27]$. In the presence of an additional symmetry $[H, U]=0,\{C, U\}=$ 0 , where $C$ is the chiral symmetry operator, $q$ becomes real also in classes AIII, CI, and DIII [28]. (ii) We use $q\left(H_{\lambda}\right)$ to predict $\left[q\left(H_{\lambda}\right)\right.$ is real $]$ or to rule out $\left[q\left(H_{\lambda}\right)\right.$ is complex $]$ robust zero-energy crossings in the respective symmetry classes. As a side product of this analysis we also derive the important result that if there are two parameters $\lambda_{1}$ and $\lambda_{2}$, robust zero energy crossings can exist in the $\left(\lambda_{1}, \lambda_{2}\right)$-parameter plane also when $q\left(H_{\lambda_{1}, \lambda_{2}}\right)$ is complex.

We complement our exact algebraic formalism with almost degenerate perturbation theory [29] applied to an eigenstate $|\psi\rangle$ of $H_{0}$ and its symmetry partner eigenstates (SPEs) $|T \psi\rangle,|P \psi\rangle$, and $|C \psi\rangle$, where $T, P$, and $C$ are the operators of time-reversal (TR), particle-hole (PH), and chiral symmetry, respectively. Using this approach in combination with a scaling argument $[24,30]$, we confirm the predicted universality of the exponent $\alpha[24,31,32]$ characterizing the asymptotic behavior of the ensemble averaged density of states $\rho \sim E^{\alpha}$ in the limit $E \rightarrow 0$ for each of the ten symmetry classes, respectively. 
This approach makes transparent why the exponent $\alpha$ does not depend on the choice of the random matrix ensemble.

In the second part of this paper (Sec. IV), we study impurity-driven topological phase transitions. We discuss the relation between the zero-energy crossings in $d=0$ and topological phase transitions in $d>0$ for systems where the topological invariant $\mathcal{Q}$ is changed by band inversion at the high-symmetry points $\boldsymbol{\Gamma}=-\boldsymbol{\Gamma}+\boldsymbol{G}$ of the Brillouin zone ( $\boldsymbol{G}$ is a reciprocal lattice vector). Our approach is to first consider a generic translationally invariant Hamiltonian $H_{0}$ and a local perturbation $\lambda H_{1}$ causing a zero-energy crossing at $\lambda=\lambda_{c}^{\infty}$. The robust zero-energy crossings are then utilized to provide an estimate for the impurity strengths necessary to drive topological phase transitions in the presence of an impurity lattice. For this purpose, we analyze $q\left(H_{\lambda}(\boldsymbol{\Gamma})\right)$, where $H_{\lambda}=H_{0}+\lambda H_{1}^{\prime}$ and $H_{1}^{\prime}$ describes the impurity lattice formed by periodically continuing $H_{1}$ with a lattice constant $a$. For each $\Gamma$, a critical value $\lambda_{\mathrm{c}}(\boldsymbol{\Gamma})$ exists, and they all coincide $\lambda_{\mathrm{c}}(\boldsymbol{\Gamma})=\lambda_{\mathrm{c}}^{\infty}$ in the limit $a / \xi^{\infty} \rightarrow \infty$, where $\xi^{\infty}$ is the decay length of the zero-energy eigenstate wave function of $H_{0}+\lambda_{\mathrm{c}}^{\infty} H_{1}$. However, at finite $a$, the $\lambda_{\mathrm{c}}(\boldsymbol{\Gamma})$ being unequal give rise to a range of values $\lambda$ where $H_{\lambda}$ is nontrivial even though $H_{0}$ is trivial. We illustrate this general behavior analytically in the context of the 1D Kitaev model [3] (equivalently the SSH model [33]).

The closing of the energy gap can also occur away from the high-symmetry points of the Brillouin zone. In this case, one can consider $\boldsymbol{k}$ as a parameter and use the zero dimensional $q\left(H_{\lambda}(\boldsymbol{k})\right)$ to analyze the phase transitions. The main difference is that the zero-dimensional Hamiltonian $H_{\lambda}(\boldsymbol{k})$, where $\boldsymbol{k}$ is considered to be a fixed parameter, usually belongs to a different symmetry class than the full higher dimensional Hamiltonian containing all the momentum blocks. We have studied the impurity lattices in several two-dimensional models, which show this kind of gap closings away from the high-symmetry points. From these closings arises a rich variety of topological phases. In particular, we demonstrate that similarly as in the case of ferromagnetic Shiba lattices [16], it is possible to obtain large Chern numbers also in $p_{x}+i p_{y}$ superconductors, but in this case one only needs nonmagnetic impurities instead of magnetic atoms. Furthermore, we use $q\left(H_{\lambda}(\boldsymbol{k})\right)$ to reveal a spiderweblike momentum space structure of the energy gap closings that separate the topologically distinct phases in $p_{x}+i p_{y}$ superconductors in the presence of an impurity lattice. Both the Shiba lattice [16] and the $p_{x}+i p_{y}$ superconductor models belong to class D in the classification table for topological insulators and superconductors [8]. However, the idea of using an impurity lattice to design topologically nontrivial phases with large Chern numbers is more general. For this purpose we demonstrate that an impurity lattice can give rise to large Chern numbers also in Chern insulators belonging to class $\mathrm{A}$ in the classification table.

\section{GENERALIZED ROOTS OF det $H$ AND RELATION TO ZERO-ENERGY CROSSINGS}

There are three types of symmetries - chiral, TR, and PH symmetry-depending on the presence or absence of which a
TABLE I. The first four columns define the symmetry classes. The absence of symmetry is denoted as " 0 " and if TR or PH symmetries are present the values of $\epsilon_{T}= \pm 1$ and $\epsilon_{P}= \pm 1$ are given. $v$ is the number of SPEs. $q(H)$ is the generalized $\nu$ th root of $\operatorname{det} H$, satisfying $|q(H)|^{\nu}=|\operatorname{det} H| . q(H)$ takes values in $\mathcal{W} . \mathcal{Q}$ is the topological invariant in $d=0 . \alpha$ is the exponent characterizing the asymptotic behavior of the ensemble averaged density of states $\rho(E) \sim|E|^{\alpha}$ in the limit $E \rightarrow 0$. In classes with chiral symmetry, in the presence of an additional symmetry $[H, U]=0,\{C, U\}=0$, the exponent $\alpha$ is lowered by one $\alpha_{U}=\alpha-1$. In classes AIII, CI, and DIII, a symmetry $U$ ensures that $q(H)$ is real up to a phase $e^{i \varphi(U)}$ which only depends on $U$. By taking this phase factor into account in the definition of $q$ (denoted $q_{U}$ in the text), $\mathcal{W}$ is reduced to $\mathcal{W}_{U}$. Moreover, $U$ enables the definition of a vector $\mathcal{Q}_{U}$ of topological invariants in these classes.

\begin{tabular}{|c|c|c|c|c|c|c|c|c|c|c|c|}
\hline & $T^{2}$ & $P^{2}$ & $C^{2}$ & $v$ & $q(H)$ & $\mathcal{W}$ & $\mathcal{Q}$ & $\alpha$ & $\mathcal{W}_{U}$ & $\mathcal{Q}_{U}$ & $\alpha_{U}$ \\
\hline AIII & 0 & 0 & 1 & 2 & $\operatorname{det} D$ & $\mathbb{C}$ & 0 & 1 & $\mathbb{R}$ & $\mathbb{Z}^{n}$ & 0 \\
\hline A & 0 & 0 & 0 & 1 & $\operatorname{det} H$ & $\mathbb{R}$ & $\mathbb{Z}$ & 0 & & & \\
\hline$\overline{\mathrm{CI}}$ & +1 & -1 & 1 & 2 & $\operatorname{det} D$ & $\mathbb{C}$ & 0 & 1 & $\mathbb{R}$ & $\mathbb{Z}^{n}$ & $\overline{0}$ \\
\hline $\mathrm{AI}$ & +1 & 0 & 0 & 1 & $\operatorname{det} H$ & $\mathbb{R}$ & $\mathbb{Z}$ & 0 & & & \\
\hline BDI & +1 & +1 & 1 & 2 & $\sqrt{\frac{(-1)^{N}}{\operatorname{det} \mathcal{P}} \operatorname{Pf}(H \mathcal{P})}$ & $\mathbb{R}$ & $\mathbb{Z}_{2}$ & 0 & $\mathbb{R}$ & $\mathbb{Z}^{n}$ & 0 \\
\hline $\mathrm{D}$ & 0 & +1 & 0 & 2 & $\sqrt{\frac{(-1)^{N}}{\operatorname{det} \mathcal{P}}} \operatorname{Pf}(H \mathcal{P})$ & $\mathbb{R}$ & $\mathbb{Z}_{2}$ & 0 & & & \\
\hline DIII & -1 & +1 & 1 & 4 & $\operatorname{Pf}(D)$ & $\mathbb{C}$ & 0 & 1 & $\mathbb{R}$ & $\left.\mathbb{Z}^{n_{1}}, \mathbb{Z}_{2}^{n_{2}}\right)$ & 0 \\
\hline AII & -1 & 0 & 0 & 2 & $\sqrt{\frac{1}{\operatorname{det} \mathcal{T}}} \operatorname{Pf}(H \mathcal{T})$ & $\mathbb{R}$ & $\mathbb{Z}$ & 0 & & & \\
\hline CII & -1 & -1 & 1 & 4 & & & 0 & 3 & & & 2 \\
\hline $\mathrm{C}$ & 0 & -1 & 0 & 2 & & & 0 & 2 & & & \\
\hline
\end{tabular}

given Hamiltonian matrix $H$ is said to belong to one of ten symmetry classes $[24,25]$ :

$$
\begin{aligned}
\text { chiral: }\{H, C\}=0, & C^{2}=1 \\
\text { TR: }[H, T]=0, & T^{2}=\epsilon_{T} \\
\text { PH: }\{H, P\}=0, & P^{2}=\epsilon_{P}
\end{aligned}
$$

where $T=\mathcal{T} K, P=\mathcal{P} K, K$ is the operator of complex conjugation, and $C, \mathcal{T}$, and $\mathcal{P}$ are unitary matrices. TR and $\mathrm{PH}$ symmetry are characterized by the $\operatorname{sign} \epsilon_{T}= \pm 1$ and $\epsilon_{P}= \pm 1$, respectively. For a list of symmetry classes (determined by the absence " 0 " or the presence of various symmetries with $\left.\epsilon_{T}= \pm 1, \epsilon_{P}= \pm 1\right)$ and an overview of results, see Table I. In the presence of a symmetry $C, T$, or $P$, one finds for each eigenstate $|\psi\rangle$ of $H$ so-called SPEs $|C \psi\rangle,|T \psi\rangle$, or $|P \psi\rangle$, respectively. Equations (1) imply that $|T \psi\rangle(|C \psi\rangle$ and $|P \psi\rangle)$ is an eigenstate of $H$ with the same (with opposite) energy as $|\psi\rangle$. However, TR symmetry with $T^{2}=1$ does not guarantee the existence of an orthogonal symmetry partner eigenstate (SPE), because the Kramers theorem applies only to the case $T^{2}=-1$. Therefore, in the present context such a symmetry should rather be understood as a constraint on $H$. The number of SPEs $v$ for all ten symmetry classes are shown in Table I.

The determinant of a matrix equals the product of its eigenvalues det $H=\prod_{i} E_{i}$. Hence, det $H$ allows us to analyze zero-energy crossings and $d=0$ topological transitions. We show that in all symmetry classes, except for $\mathrm{C}$ and CII, one can explicitly define a function $q(H)$ with two key properties:

(i) $q(H)$ is a (complex valued) polynomial expression in the matrix elements $H_{i j}$ of the Hamiltonian. 
(ii) $q(H)$ satisfies $|\operatorname{det} H|=|q(H)|^{\nu}$, where $v$ is the number of SPEs in the respective symmetry class.

Property (ii) defines in which sense it is justified to call $q(H)$ the (generalized) $\nu$ th root of $\operatorname{det} H$. However, property (i) constitutes the important difference between $q(H)$ and $\sqrt[v]{\operatorname{det} H}$. Most importantly, $q(H)$ shares with $\operatorname{det} H$ the property that it vanishes if and only if the Hamiltonian has a zero eigenvalue. Before defining $q$ in Sec. II A, we now explain its relation to the $d=0$ topological invariants. Its relation to zero-energy crossings is detailed in Sec. II B.

From $|\operatorname{det} H|=|q(H)|^{\nu}$ and the possibility to calculate $\operatorname{det} H=\prod_{i} E_{i}$ as the product of the Hamiltonian's eigenvalues follows

$$
q(H)=\prod_{i}^{\prime} E_{i},
$$

when $q$ is real. Here, the prime indicates that only one member of the set of $v$ SPEs contributes a factor $E_{i}$. In principle, $q$ is only defined up to a sign, but once the sign is chosen, it remains well defined when changing $H$ adiabatically. In consequence, we find that in those symmetry classes where the $d=0$ invariant $\mathcal{Q}$ exists, $q$ changes sign when $\mathcal{Q}$ changes by \pm 1 . On the other hand, in the presence of an additional symmetry $U$ in classes AIII, CI, and DIII, $H$ is block diagonal with blocks $H_{ \pm i}(i=1,2, \ldots, n)$, and if $\{C, U\}=0$ an invariant $\mathcal{Q}_{H_{ \pm i}}$ exists for each block; cf. Appendix A. Then, $q$ changes sign when one of the $\mathcal{Q}_{H_{+i}}$ changes by \pm 1 . Formally, this corresponds to

$$
\operatorname{sgn} q(H)= \begin{cases}(-1)^{\mathcal{Q}} & \text { A, AI, AII, BDI, D } \\ \prod_{i=1}^{n}(-1)^{\mathcal{Q}_{H_{i}}} & \mathrm{AIII}+U, \mathrm{CI}+U, \mathrm{DIII}+U,\end{cases}
$$

where $X+U$ means "for Hamiltonians from symmetry class $X$ with the additional symmetry $U,\{C, U\}=0$." For examples of unitary symmetries in the context of topological order see Refs. [28] and [34-41].

\section{A. Defining generalized roots of $\operatorname{det} H$}

Classes $A$ and AI. Since SPEs are absent, we can define $q(H)=\operatorname{det} H$.

Classes BDI and D. Hamiltonians in these symmetry classes obey $\mathrm{PH}$ symmetry with $P^{2}=+1$. In accordance with Refs. [3,27,42], we define the real polynomial $q(H)=$ $\sqrt{(-1)^{N} / \operatorname{det} \mathcal{P}} \operatorname{Pf}(H \mathcal{P})$, where $H$ is a $2 N \times 2 N$ matrix. Using $(\mathcal{P} K)^{2}=\mathcal{P} \mathcal{P}^{\dagger}=+1$ and Eq. (1c) it follows that $\mathcal{P}=$ $\mathcal{P}^{T}$ and $H \mathcal{P}=-(H \mathcal{P})^{T}$. Thus $\operatorname{Pf}(H \mathcal{P})$ is well defined. From the general property of the $\operatorname{Pfaffian} \operatorname{Pf}(A)^{2}=\operatorname{det}(A)$, we then infer $|q(H)|^{2}=|\operatorname{det} H|$.

Class AII. Hamiltonians in AII obey TR symmetry with $T^{2}=-1$. We proceed analogously to the derivation of $q(H)$ for classes BDI and $\mathrm{D}$ and thus define the real polynomial $q(H)=\sqrt{1 / \operatorname{det} \mathcal{T}} \operatorname{Pf}(H \mathcal{T})$.

Classes AIII and CI. Hamiltonians in these classes have a chiral symmetry. We focus on the case where $\operatorname{tr} C=0$ [43]. This allows us to choose a basis such that

$$
\begin{aligned}
C & =\left(\begin{array}{cc}
\mathbf{1}_{N} & \mathbf{0}_{N} \\
\mathbf{0}_{N} & -\mathbf{1}_{N}
\end{array}\right), \\
H & =\left(\begin{array}{cc}
\mathbf{0}_{N} & D \\
D^{\dagger} & \mathbf{0}_{N}
\end{array}\right) .
\end{aligned}
$$

Since $|\operatorname{det} H|=|\operatorname{det} D|^{2}$ we can straightforwardly define the complex polynomial $q(H)=\operatorname{det} D$.

Chiral symmetry alone does not put any restriction on $D$. Albeit TR symmetry restricts $D$ in class CI, the phase of $\operatorname{det} D$ does depend on the parameters of the Hamiltonian in both classes, CI and AIII. In the following, we show that the phase of det $D$ becomes independent of the Hamiltonian's parameters if there is a symmetry $U$ satisfying $[H, U]=\{C, U\}=0$. In the basis of Eq. (4a), $\{C, U\}=0$ implies

$$
U=\left(\begin{array}{ll}
\mathbf{0}_{N} & U_{1} \\
U_{2} & \mathbf{0}_{N}
\end{array}\right)
$$

with unitary matrices $U_{1}$ and $U_{2}$. The symmetry $[H, U]=0$ implies $U_{1} D^{\dagger}=D U_{2}$. This can be used to show $(\operatorname{det} D)^{*}=$ $\operatorname{det}\left(U_{1}^{\dagger} U_{2}\right) \operatorname{det} D$, which means that the complex phase of $q(H)$ is independent of $H$ in the presence of the additional symmetry $U$. Hence, we define the real polynomial $q_{U}(H)=$ $\sqrt{\operatorname{det}\left(U_{1}^{\dagger} U_{2}\right)} \operatorname{det} D$.

Class DIII. A polynomial expression for the fourth root of det $H$ is a central result of Ref. [28]. For completeness, we briefly summarize the derivation here. Due to chiral symmetry, $H$ is of off-diagonal form, cf. Eq. (4b). TR symmetry with $T^{2}=-1$ renders $D=-D^{T}$ antisymmetric. This enables the definition $q(H)=\operatorname{Pf} D$. Also in class DIII, the complex phase of $q(H)$ loses its dependence on the Hamiltonian's parameters in the presence of a symmetry $[H, U]=\{C, U\}=0$. Then, the real polynomial $q_{U}(H)=\sqrt{(-1)^{N} / \operatorname{det} W}$ Pf $D$ can be defined, where $W$ is a block of $U=\left(\begin{array}{cc}0 & W \\ W^{*} & 0\end{array}\right)$ in the basis where $C$ is diagonal, cf. Eq. (4a).

Classes $C$ and CII. In these classes, it is not possible to define $q(H)$ as a polynomial in the matrix elements of $H$. For a justification, see Appendix B.

\section{B. Relation to zero-energy crossings}

Since $|q(H)|^{v}=|\operatorname{det} H|$, the zeros of $q(H)$ coincide with the zeros of det $H$ and the existence of zero-energy eigenstates of $H$. For predicting zero-energy crossings, $q(H)$ is more useful than det $H$ because (i) it is a complex number in classes AIII, CI, and DIII and thus has a phase degree of freedom and (ii) it accounts for symmetries and thus is a polynomial of lower degree than $\operatorname{det} H$. In Sec. III we supplement the following abstract discussion with additional more intuitive arguments.

In Ref. [28], the possibility to predict zero-energy crossings using $q(H)$ was illustrated for a Hamiltonian $H_{\lambda}=H_{0}+\lambda H_{1}$, where $H_{0}$ is from DIII and $\lambda H_{1}$ is an on-site potential with impurity strength $\lambda$. While det $H_{\lambda}$ is a real fourth order polynomial in $\lambda, q\left(H_{\lambda}\right)$ is a complex first order polynomial. Consequently, $q\left(H_{\lambda}\right)$ as a function of $\lambda$ takes values on a straight line in the complex plane which generically does not contain the origin, so that zero-energy eigenstates will not occur for any $\lambda$; cf. solid line in Fig. 1(a). More generally, $q(H)$ describes a continuous curve in the complex plane under arbitrary continuous parameter changes of $H$ and will not go through the origin, so that it will not give rise to zero-energy states without fine tuning; cf. dashed line in Fig. 1(a). As a result, in classes AIII, CI, and DIII, where $q(H) \in \mathbb{C}$, changing a single parameter in $H$ will not induce robust zero-energy crossings. 


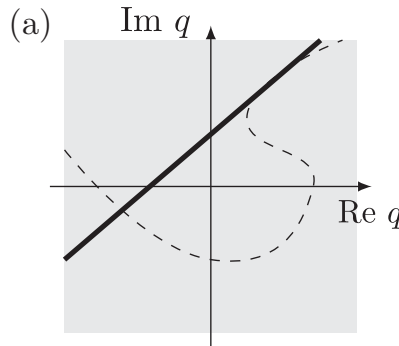

AlII, Cl, DIII (b)

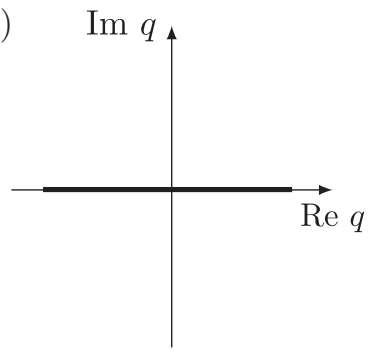

A, Al, All, BDI, D

$[\mathrm{AlII}+U, \mathrm{Cl}+U, \mathrm{DIII}+U]$

FIG. 1. Illustration of the real and imaginary parts of $q\left(H_{\lambda}\right)$ as a function of an external parameter $\lambda$. (a) In classes AIII, CI, and DIII, $q\left(H_{\lambda}\right)$ takes complex values and generically does not become zero, independently of whether a parameter is considered on which $q$ depends linearly (solid line) or with some higher power (dashed line). (b) In classes A, AI, AII, BDI, and D [AIII, CI, and DIII with additional symmetry $U$ which anticommutes with $C] q\left(H_{\lambda}\right)\left[q_{U}\left(H_{\lambda}\right)\right]$ is always real. In these cases the change of sign of $q\left(H_{\lambda}\right)$ as a function of $\lambda$ guarantees the existence of robust zero-energy crossing at some value $\lambda=\lambda_{c}$.

Consider now the case where $q(H)$ is real. Focusing on $H_{\lambda}=H_{0}+\lambda H_{1}$, where $H_{1}$ is an arbitrary local perturbation, we find $q\left(H_{\lambda}\right)=\sum_{i=0}^{n} b_{i} \lambda^{i}$, where $b_{i} \in \mathbb{R}$ and $n$ depends on $H_{1}$. Clearly, when $n$ is odd, $q\left(H_{\lambda}\right)$ crosses zero at least once upon tuning $\lambda$ from $-\infty$ to $+\infty$ [Fig. 1(b)]. For even $n$, an even number of zero-energy crossings will occur, including the possibility that $q$ does not cross zero.

Some remarks are in order. (i) Although the zero-energy crossings are most easily analyzed as a function of $\lambda$, they appear also as a function of $H_{0}$ bulk parameters like the chemical potential. This is illustrated in Fig. 2, where we plot the critical impurity strength $\lambda_{\mathrm{c}}^{\infty}$ corresponding to a zero-energy crossing for an impurity $\lambda H_{1}=\lambda c_{0}^{\dagger} c_{0}$, where $c_{i}$ annihilates a spinless electron on site $i$, in an infinite $p_{x}+i p_{y}$ superconductor. The unperturbed Hamiltonian in momentum space reads

$$
H_{0}(\boldsymbol{k})=\frac{1}{2} \sum_{\boldsymbol{k}}\left(c_{\boldsymbol{k}}^{\dagger}, c_{-\boldsymbol{k}}\right)\left(\begin{array}{cc}
h(\boldsymbol{k}) & \Delta(\boldsymbol{k}) \\
\Delta(\boldsymbol{k})^{*} & -h(\boldsymbol{k})
\end{array}\right)\left(\begin{array}{c}
c_{\boldsymbol{k}} \\
c_{-\boldsymbol{k}}^{\dagger}
\end{array}\right)
$$

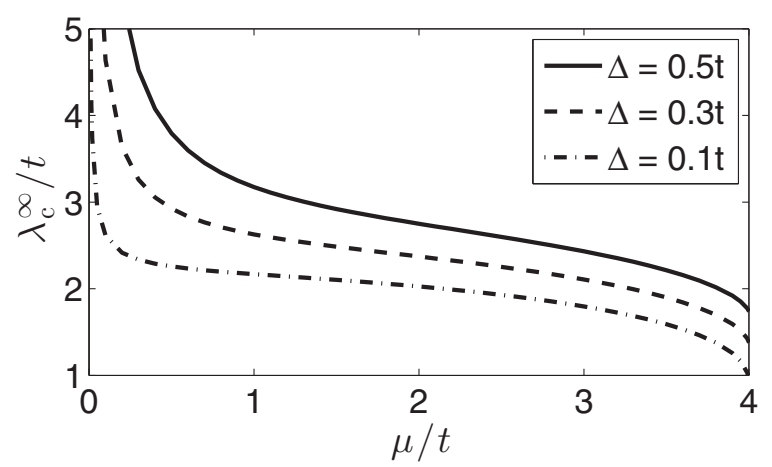

FIG. 2. The critical impurity strength $\lambda_{\mathrm{c}}^{\infty}$ of an on-site potential impurity $\lambda H_{1}=\lambda c_{0}^{\dagger} c_{0}$ in a $p_{x}+i p_{y}$ superconductor depends on the chemical potential $\mu$. Hence, zero-energy crossing appear not only by tuning $\lambda$, but also by tuning $\mu$.

with

$$
\begin{aligned}
h(\boldsymbol{k}) & =-2 t\left(\cos k_{x}+\cos k_{y}\right)-\mu, \\
\Delta(\boldsymbol{k}) & =\Delta\left(\sin k_{x}+i \sin k_{y}\right) .
\end{aligned}
$$

Here $t$ and $\Delta$ parametrize the nearest neighbor hopping and superconducting pairing, respectively, and $\mu$ is the chemical potential. $\lambda_{\mathrm{c}}^{\infty}$ was determined by performing a $T$-matrix calculation [44]. (ii) In classes AIII, CI, and DIII the presence of a symmetry $U$ is not a necessary but a sufficient condition to render the phase of $q(H)$ independent of $H$ [28]. In fact, contrary to the set of equations $[H, U]=0$, the necessary and sufficient condition $\operatorname{Im} q=\tan \varphi \operatorname{Re} q$ is just a single equation. (iii) The argument for the existence of zero-energy crossings when $q$ is real can be generalized to interacting models where $H_{0}$ needs to be calculated self-consistently when the external parameter $\lambda$ is varied. Assuming that the coefficients $b_{i}$ become continuous functions of $\lambda$ and there is no spontaneous symmetry breaking, the change of sign of $q\left(H_{\lambda}\right)$ still guarantees the existence of a robust zero-energy crossing. (iv) If $q\left(H_{\lambda}\right) \in \mathbb{C}$ it can still be used to predict robust zero-energy crossings. However, in this case one needs at least two external parameters $\left(\lambda_{1}, \lambda_{2}\right)$, and the robust crossings can only be guaranteed to occur at particular critical points $\left(\lambda_{1 c}, \lambda_{2 c}\right)$ within a parameter plane. Namely, consider the real and imaginary parts of $q\left(H_{\lambda_{1}, \lambda_{2}}\right)$ as a function of an external parameter $\lambda_{1}$ for two fixed values of another external parameter $\lambda_{2}=\lambda_{2 a}$ and $\lambda_{2}=\lambda_{2 b}$. If the two curves are on opposite sides of the origin as illustrated in Fig. 3, under certain assumptions they cannot be smoothly deformed into each other unless $q\left(H_{\lambda_{1}, \lambda_{2}}\right)=0$ at a particular point $\lambda_{1}=\lambda_{1 c}$ and $\lambda_{2}=\lambda_{2 c}$. Therefore this kind of situation typically leads to the existence of a robust zero-energy crossing at a specific point within the $\left(\lambda_{1}, \lambda_{2}\right)$-parameter plane.

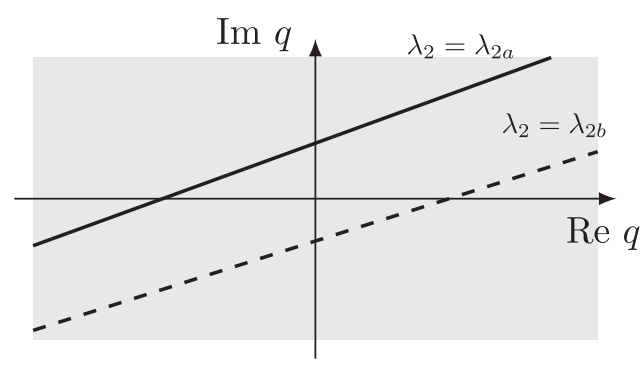

FIG. 3. Illustration of the real and imaginary parts of $q\left(H_{\lambda_{1}, \lambda_{2}}\right)$ as a function of an external parameter $\lambda_{1}$ for two fixed values of another external parameter $\lambda_{2}=\lambda_{2 a}$ and $\lambda_{2}=\lambda_{2 b}$ in classes where $q(H)$ takes complex values. If the two curves are on opposite sides of the origin as illustrated in the figure and assuming that for $\lambda_{2} \in\left[\lambda_{2 a}, \lambda_{2 b}\right] \operatorname{Re} q$ (or $\operatorname{Im} q$ ) is a continuously increasing/decreasing function of $\lambda$ with $\min [\operatorname{Re} q]<0$ and $\max [\operatorname{Re} q]>0(\min [\operatorname{Im} q]<0$ and $\max [\operatorname{Im} q]>0)$, these curves cannot be smoothly deformed into each other except if $q\left(H_{\lambda_{1}, \lambda_{2}}\right)=0$ at a particular point $\lambda_{1}=\lambda_{1 c}$ and $\lambda_{2}=\lambda_{2 c}$. Therefore there exists a robust zero-energy crossing within the $\left(\lambda_{1}, \lambda_{2}\right)$-parameter plane. 


\section{LEVEL REPULSION AND DENSITY OF STATES IN RANDOM MATRIX ENSEMBLES}

There are relations between $q(H)$ and the allowed couplings of SPEs as well as the density of states in random matrix ensembles. These relations are the subject of this section.

We focus on the eigenstate $|\psi\rangle$ of a Hamiltonian $H_{0}$ which, together with its SPEs, is the one with energy closest to zero. Subsequently, we study how the energy corresponding to this set of SPEs evolves as the strength of a perturbation $H_{1}$ from the same symmetry class as $H_{0}$ is tuned. Therefore, we employ almost degenerate perturbation theory [29] in the subspace spanned by these SPEs. Moreover, we consider ensembles of random matrices from any of the ten symmetry classes. Then, by adopting a scaling argument from Ref. [30], we use our perturbative results to derive the universal asymptotic behavior of the respective ensemble averaged density of states in the zero-energy limit.

As an immediate consequence of the defining symmetry properties Eqs. (1), the matrix elements describing the coupling of SPEs are restricted as follows:

$$
\begin{aligned}
& \left\langle\psi\left|H_{1}\right| C \psi\right\rangle=-\left\langle\psi\left|H_{1}\right| C \psi\right\rangle^{*}, \\
& \left\langle\psi\left|H_{1}\right| T \psi\right\rangle=\epsilon_{T}\left\langle\psi\left|H_{1}\right| T \psi\right\rangle, \\
& \left\langle\psi\left|H_{1}\right| P \psi\right\rangle=-\epsilon_{P}\left\langle\psi\left|H_{1}\right| P \psi\right\rangle .
\end{aligned}
$$

The possible couplings, restricted by these conditions, are depicted in Fig. 4 for all ten symmetry classes. Assuming that the energy $\epsilon$ of $|\psi\rangle$ is sufficiently close to zero, the effect of a generic perturbation $H_{1}$ from the same symmetry class as $H_{0}$ on $|\psi\rangle$ and its SPEs can be calculated via almost degenerate perturbation theory [29]. The energies of the set of almost degenerate states in the presence of the perturbation is obtained from the eigenvalues of $\bar{H}=\Pi_{|\psi\rangle}\left(H_{0}+H_{1}\right) \Pi_{|\psi\rangle}$, where $\Pi_{|\psi\rangle}$ projects onto the subspace spanned by $|\psi\rangle$ and its SPEs.

As an example we consider symmetry class AIII, where the matrix elements for the projected perturbation read $\left\langle\psi\left|H_{1}\right| \psi\right\rangle=-\left\langle C \psi\left|H_{1}\right| C \psi\right\rangle=\epsilon_{1}$, and $\left\langle\psi\left|H_{1}\right| C \psi\right\rangle=i \epsilon_{2}$; $\epsilon_{1}, \epsilon_{2} \in \mathbb{R}$. Hence, we find in the basis $(|\psi\rangle,|C \psi\rangle)$

$$
\begin{aligned}
\bar{H}^{\mathrm{AIII}} & =\left(\begin{array}{cc}
\epsilon+\epsilon_{1} & i \epsilon_{2} \\
-i \epsilon_{2} & -\epsilon-\epsilon_{1}
\end{array}\right), \\
E_{ \pm}^{\mathrm{AIII}} & = \pm \sqrt{\left(\epsilon+\epsilon_{1}\right)^{2}+\epsilon_{2}^{2}} .
\end{aligned}
$$

Because $|\psi\rangle$ couples to its chiral partner, one generically expects an avoided level crossing upon tuning the perturbation.

Exploiting the symmetry constraints [Eqs. (8)], one can derive analogous results for all ten symmetry classes. The corresponding energies are always of the form $E_{+}=$ $\sqrt{\left(\epsilon+\epsilon_{1}\right)^{2}+\epsilon_{2}^{2}+\cdots+\epsilon_{\alpha+1}^{2}}$ with $\alpha=0,1,2$, or 3 depending on the symmetry class considered. The corresponding values of $\alpha$ for all ten symmetry classes are shown in Table I, and as we show below $\alpha$ is the universal exponent determining the low-energy asymptotic behavior of the ensemble averaged density of states. $\alpha=0$ is to be understood as $E_{+}=\epsilon+\epsilon_{1}$. $E_{+}$is twice degenerate in classes AII, DIII, and CII. In those classes with a $\mathrm{PH}$ symmetric spectrum one also gets $E_{-}=-E_{+}$.

We now consider arbitrary random matrix ensembles from any of the ten symmetry classes. Our perturbative results can then be used to calculate the universal asymptotic behavior of the respective ensemble averaged density of states $\rho(E)$ in the limit $E \rightarrow 0$ [30]. To this end, we consider the sum $H_{0}+H_{1}$ to be a realization of a random matrix from the respective ensemble. The $\epsilon_{i}$ are then random variables with the corresponding probability distribution $W(\boldsymbol{\epsilon}), \boldsymbol{\epsilon}=$ $\left(\epsilon_{1}, \ldots, \epsilon_{\alpha+1}\right)$, which we do not have to know in detail. $\epsilon$ is not independent of $\epsilon_{1}$ and thus has been absorbed in $\epsilon_{1}$. The ensemble averaged density of states reads

$$
\rho(E)=\int \mathrm{d}^{(\alpha+1)} \epsilon W(\boldsymbol{\epsilon}) \delta\left(|E|-\sqrt{\epsilon^{2}}\right) .
$$

Upon rescaling $\boldsymbol{\epsilon} \mapsto|E| \boldsymbol{\epsilon}$, we obtain $\rho(E)=|E|^{\alpha} \int$ $\mathrm{d}^{(\alpha+1)} \epsilon W(|E| \epsilon) \delta\left(1-\sqrt{\epsilon^{2}}\right)$. The symmetry constraints for each symmetry class were already taken into account in the Hamiltonian $\bar{H}$ and $\alpha$, respectively, so that generically $W(|E| \boldsymbol{\epsilon})$ will remain finite in the limit $|E| \rightarrow 0$. Hence, for energies less than the average level spacing $\delta_{0}$, we obtain the asymptotic behavior

$$
\rho(E) \sim|E|^{\alpha} \quad|E| \lesssim \delta_{0} .
$$

The exponents $\alpha$ that result from this argument are listed in Table I and agree with those stated in Refs. [24,31,32]. $\alpha$ relates to $q(H)$ in the following way: $\alpha>1$ in classes $\mathrm{C}$ and CII where $q$ cannot be defined. $\alpha=1$ in classes AIII, CI, and DIII where $q \in \mathbb{C}$. $\alpha=0$ when $q$ is real. Finally, when a matrix ensemble with chiral symmetry is restricted to a subset of matrices $H$ satisfying $[H, U]=\{C, U\}=0$, the matrix element $\left\langle\psi\left|H_{1}\right| C \psi\right\rangle$ vanishes and $\alpha$ is reduced to $\alpha_{U}=\alpha-1$; cf. Table. I.

\section{IMPURITY-DRIVEN TOPOLOGICAL PHASE TRANSITIONS}

In this section we consider the topological order of a translationally invariant Hamiltonian $H=H_{0}+\lambda H_{1}^{\prime}$, where $H_{0}$ is gapped and $H_{1}^{\prime}$ describes a lattice of impurities with period $a$ determining the overall translational symmetry of

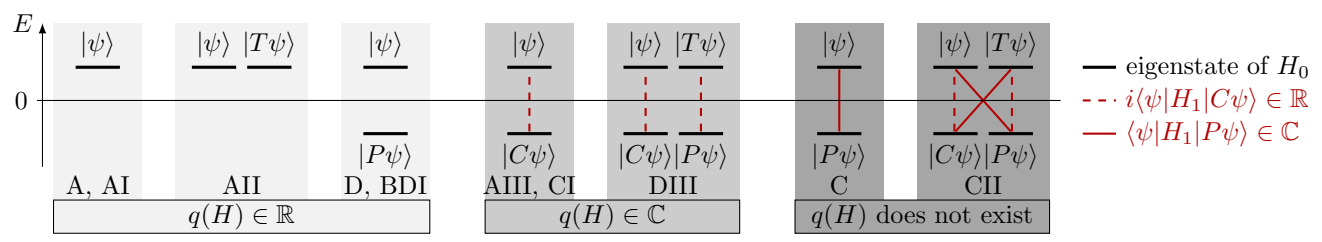

FIG. 4. For each symmetry class, an eigenstate $|\psi\rangle$ of $H_{0}$ and its SPEs are shown. A perturbation $H_{1}$ from the same class as $H_{0}$ couples SPEs with opposite energies as indicated in the figure. The possible couplings to chiral (PH) partners are shown by dashed (solid) red lines. The coupling to the chiral SPE vanishes when the Hamiltonian obeys an additional symmetry $U$ with $\{C, U\}=0$. 
the system. A general translationally invariant Hamiltonian in dimension $d>0$ is block diagonal in momentum space, $H=\bigoplus_{k} H(\boldsymbol{k})$ [45]. Each Hermitian block $H(\boldsymbol{k})$ describes the physics of a $d=0$ system. In particular, there are $2^{d}$ high symmetry points $\Gamma$ in the Brillouin zone, which satisfy $\boldsymbol{\Gamma}=-\boldsymbol{\Gamma}+\boldsymbol{G}$ with $\boldsymbol{G}$ a reciprocal lattice vector, and each $d=0$ Hamiltonian $H(\boldsymbol{\Gamma})$ belongs to the same symmetry class as $H$ itself. Hence, we can calculate $q(H(\Gamma))$ [using the definition of $q(H)$ for the symmetry class of $H$ itself] to determine zero-energy crossings of $H(\boldsymbol{\Gamma})$.

In the case of the impurity lattice, the blocks in momentum space become $H_{\lambda}(\boldsymbol{k})=H_{0}(\boldsymbol{k})+\lambda H_{1}^{\prime}(\boldsymbol{k})$. Finding the zeros $\lambda_{\mathrm{c}}(\boldsymbol{\Gamma})$ of $q\left(H_{\lambda}(\boldsymbol{\Gamma})\right)$ yields information about band inversions at $\Gamma$ which often affect the topological invariants of the system. Let us consider the case that $H_{0}+\lambda_{\mathrm{c}}^{\infty} H_{1}$ has a zero-energy eigenstate, where $H_{1}$ is a single impurity of the set of impurities forming $H_{1}^{\prime}$. Its wave function decays on a length scale $\xi^{\infty}$ because $\lambda H_{1}$ is a local perturbation and $H_{0}$ is gapped. Consider now $H_{\lambda}(\boldsymbol{\Gamma})$ in the limit $a \gg \xi^{\infty}$. Then, the boundaries of the unit cell have a vanishing effect such that

$$
\lim _{a / \xi^{\infty} \rightarrow \infty} \lambda_{c}(\Gamma)=\lambda_{c}^{\infty},
$$

independent of $\boldsymbol{\Gamma}$. However, at finite $a$ the $\lambda_{\mathrm{c}}(\boldsymbol{\Gamma})$ being unequal give rise to a range of values $\lambda$ where $H_{\lambda}$ is nontrivial even though $H_{0}$ is trivial. In systems with sufficiently dilute impurity lattice (compared to $\lambda_{\mathrm{c}}^{\infty}$ ), impurity strengths of the order of $\lambda_{c}^{\infty}$ are nevertheless needed to drive a topological phase transition. Below, we employ the 1D Kitaev model to analytically illustrate this general behavior.

The closing of the energy gap can also occur away from the high-symmetry points of the Brillouin zone. In general, this can happen at both $\boldsymbol{k}$ points distinguished by unitary symmetries of the Hamiltonian [35,36] as well as arbitrary $\boldsymbol{k}$ points, cf. Sec. IV B. Then, by considering $\boldsymbol{k}$ as a parameter, one can still use the zero-dimensional $q\left(H_{\lambda}(\boldsymbol{k})\right)$ to analyze the phase transitions. The main difference is that the zerodimensional Hamiltonian $H_{\lambda}(\boldsymbol{k})$, where $\boldsymbol{k}$ is considered as a fixed parameter, usually belongs to a different symmetry class than the full higher dimensional Hamiltonian containing all the momentum blocks. We demonstrate that similar to the case of ferromagnetic Shiba lattices [16], it is possible to obtain large Chern numbers in $p_{x}+i p_{y}$ superconductors, but in this case one only needs nonmagnetic impurities instead of the magnetic atoms. Both of these models belong to symmetry class D. Moreover, we demonstrate that an impurity lattice can give rise to large topological invariants in Chern insulators belonging to symmetry class A.

\section{A. Impurity-driven topological phase transitions in the Kitaev chain}

The general ideas presented so far can nicely be illustrated in the context of the Kitaev model [3] describing a superconductor of spinless fermions in $d=1$

$$
H_{0}=\sum_{i}\left(-t c_{i}^{\dagger} c_{i+1}+\Delta c_{i}^{\dagger} c_{i+1}^{\dagger}+\text { H.c. }\right)-\mu \sum_{i} c_{i}^{\dagger} c_{i} .
$$

To simplify the analysis we focus on $\Delta=t \in \mathbb{R}$. The Hamiltonian $H_{0}$ belongs to class BDI with artificial TR symmetry $T=K$ in addition to the intrinsic $\mathrm{PH}$ symmetry

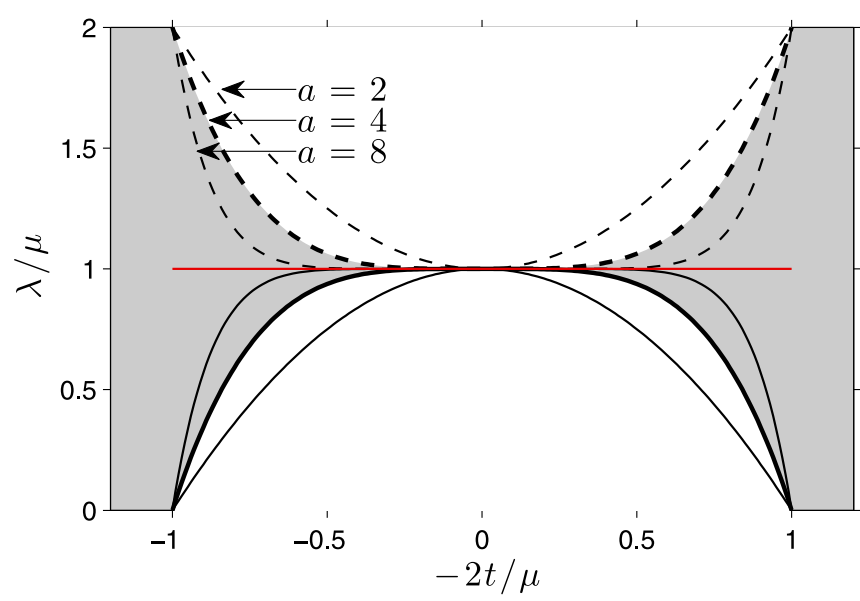

FIG. 5. Phase diagrams of the Kitaev chain in the presence of impurity lattices as a function of impurity strength $\lambda$ and $2 t / \mu$ for $\Delta=t$ and different lattice constants $a$. Gray (white) areas denote topologically nontrivial (trivial) phases in case of impurity lattice constant $a=4$. Solid (dashed) lines are $\lambda_{\mathrm{c}}(0)\left[\lambda_{\mathrm{c}}(\pi)\right]$. Solid red line is $\lambda_{\mathrm{c}}^{\infty}$.

of Bogoliubov-de Gennes Hamiltonians. For $|2 t / \mu|<1$ the system is in a trivial phase, and for $|2 t / \mu|>1$ the system supports Majorana end modes. Thus, there is a topological phase transition at $|2 t / \mu|=1$.

We now consider the effect of an impurity lattice $\lambda H_{1}^{\prime}=$ $\lambda \sum_{i} c_{a i}^{\dagger} c_{a i}$ on the topology assuming that we start from inside the topologically trivial phase $|2 t / \mu|<1$. Here, $a$ is the lattice period. Band inversions at the high symmetry $k$ points $\Gamma=$ $0, \pi$ change the parity of the topological invariant [46]. By determining the zeros of $q\left(H_{\lambda}(\Gamma)\right)$, we find that the critical

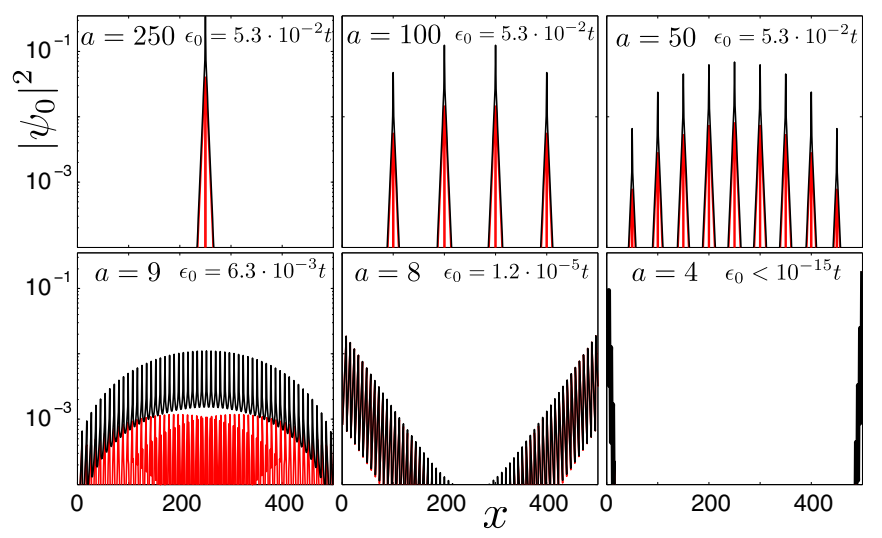

FIG. 6. Illustration of the dimensional crossover in the Kitaev chain. A single impurity introduces a single subgap state (first panel). Upon increasing the density of impurities (decreasing $a$ ) the wave functions for the impurity states start to overlap and one forms an impurity band. The topology of this band changes and protected end states form, when the impurity concentration exceeds its critical value $\left[a<9\right.$; cf. Eq. (15)]. $\psi_{0}$ is the wave function of the eigenstate with smallest positive energy $\epsilon_{0}$. Black (red) curves correspond to the particle (hole) component of $\psi_{0}$. Parameters: $2 t / \mu=0.8$, $\lambda / \mu=0.85$; cf. Fig. 5 . 
impurity strengths are (see Appendix C)

$$
\frac{\lambda_{\mathrm{c}}(\Gamma)}{\mu}=1-e^{i \Gamma}\left(\frac{-2 t}{\mu}\right)^{a}, \quad \Gamma=0, \pi
$$

with a wave function decaying on the scale $\xi^{\infty}=1 / \ln |\mu / 2 t|$. When $a \gg \xi^{\infty}$, we get $\lambda_{\mathrm{c}}(\boldsymbol{\Gamma})=\lambda_{\mathrm{c}}^{\infty}=\mu$ in agreement with Eq. (13). The phase diagrams for different values of $a$ are shown in Fig. 5. For all values of $a$, it is possible to turn the trivial phase to a topologically nontrivial phase with the help of impurity lattice. However for $a \gg \xi^{\infty}$ the trivial phase becomes nontrivial only in a very small region of parameters around $\lambda \approx \lambda_{\mathrm{c}}^{\infty}$.

As illustrated in Fig. 6, our results allow the following appealing interpretation: A single local impurity can cause a $d=0$ topological transition. Taking several $d=0$ systems with an impurity of strength $\lambda \approx \lambda_{\mathrm{c}}^{\infty}$ and coupling them together via hopping (and pairing) terms yields a system of dimensionality $d>0$ with good chances to be topologically different from the system one would obtain without impurities.

\section{B. Impurity-driven topological phase transitions in $p_{x}+i p_{y}$ superconductors}

In this section, we consider the two-dimensional $p_{x}+i p_{y}$ superconductor described by the Hamiltonian $H_{0}$ given by Eq. (6). Since $H_{0}$ belongs to class $\mathrm{D}$, there exists a $\mathbb{Z}$ topological invariant, namely the sum $C$ of all Chern numbers of the occupied bands. The topologically distinct phases of $H_{0}$ are $C=+1$ for $0<\mu / t<4, C=-1$ for $-4<\mu / t<0$, and $C=0$ for $|\mu / t|>4$. We will consider this type of superconductor in the presence of impurities described by the Hamiltonian $\lambda H_{1}=\lambda c_{0}^{\dagger} c_{0}$ arranged on a square lattice with period $a$ to form a periodic perturbation $\lambda H_{1}^{\prime}$. We demonstrate that the impurity lattice allows us to access extremely large

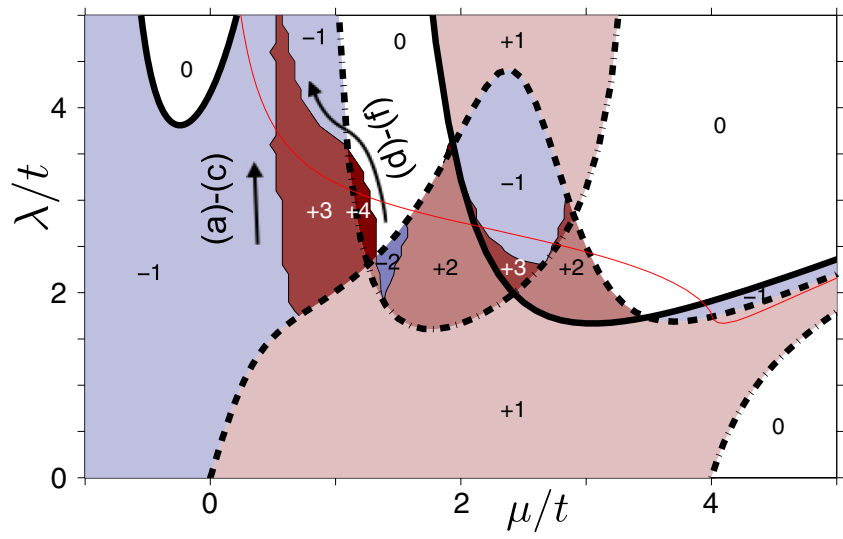

FIG. 7. Phase diagram of the $p_{x}+i p_{y}$ superconductor in the presence of an impurity lattice as a function of impurity strength $\lambda$ and chemical potential $\mu$ for $\Delta=0.5 t$ and $a=3$. Thick lines are gap closings at the high symmetry points [solid: $(0,0)$, dashed: $(0, \pi)$ and $(\pi, 0)$, dash-dot: $(\pi, \pi)]$. At thin lines, the gap closes away from the high-symmetry points. Numbers denote the sum of Chern numbers of occupied bands in the respective phase. The thin red line is $\lambda_{c}^{\infty}$. The positions of the gap closings in the momentum space for the thin lines denoted as (a)-(c) and (d)-(f) are illustrated in Fig. 8.
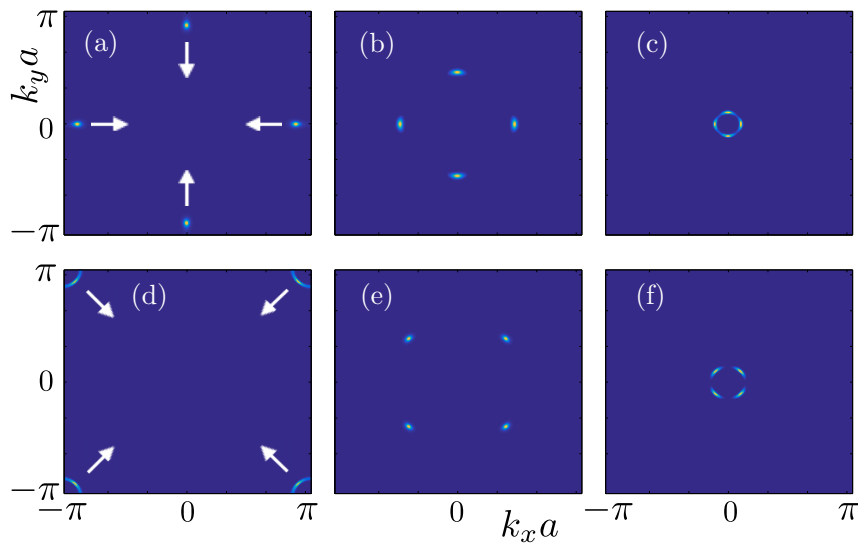

FIG. 8. Chern curvature of the impurity band for different points in the phase diagram in Fig. 7: (a) $\mu=0.6 t, \lambda=1.75 t$, (b) $\mu=0.5 t, \lambda=4 t$, (c) $\mu=0.48 t, \lambda=6.8 t$, (d) $\mu=1.37 t, \lambda=2 t$, (e) $\mu=0.85 t, \lambda=4 t$ and (f) $\mu=0.5 t, \lambda=6.8 t$. The gap closings associated with topological phase transitions give rise to momentum space topological defects, which show up as an enhanced Chern curvature in the vicinity of the gap closing point in the momentum space.

Chern numbers similarly as in the case of ferromagnetic Shiba lattices [16].

Figure 7 shows the phase diagram of the Hamiltonian $H=H_{0}+\lambda H_{1}^{\prime}$ as a function of $\lambda$ and $\mu$ for $\Delta=0.5 t$ and $a=3$ [47]. There exists a rich variety of phases, which can be accessed by tuning these parameters. The topological phase

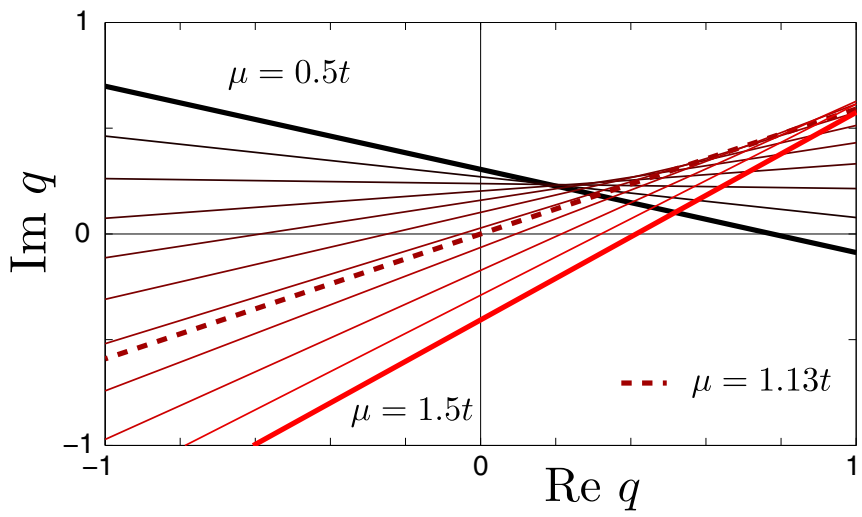

FIG. 9. Real and imaginary parts of $q\left(H_{\lambda}(\boldsymbol{k})\right)=\operatorname{det} D_{\lambda}(\boldsymbol{k})$ as a function of $\lambda$ for $k_{x} a=k_{y} a=\pi / 2, \Delta=0.5 t, a=3$, and $\mu \in$ $[0.5 t, 1.5 t]$. The lines corresponding to $\mu=0.5 t$ and $\mu=1.5 t$ are on different sides of the origin, so that the Hamiltonian realizes the situation illustrated in Fig. 3. Namely, by tuning $\mu \in[0.5 t, 1.5 t]$ one smoothly deforms the curve so that there exists a particular point $\mu=\mu_{c}$ and $\lambda=\lambda_{c}$ where $q\left(H_{\lambda}(\boldsymbol{k})\right)=0$. This leads to a gap closing at $\mu=\mu_{c}=1.13 t$ and $\lambda=\lambda_{c}=3.47 t$ explaining one point in the topological phase-transition line denoted (d)-(f) in Fig. 7. The whole phase-transition line is obtained by repeating this analysis for all $k_{x}=k_{y}=k$, where $k a \in[0, \pi]$. The unitary transformation $V$ block off-diagonalizing the Hamiltonian is not unique. However, $q\left(H_{\lambda}(\boldsymbol{k})\right)$ is unique up to an overall phase, and therefore the different choices of $V$ lead to the same figure up to the rotations of the $\operatorname{Re} q$ and $\operatorname{Im} q$ axis. 
transitions separating these phases come in two variants. First, there are transitions accompanied by a gap closing at the high symmetry points where $k_{x}=0, \pi$ and $k_{y}=0, \pi$. The corresponding phase transition lines $\lambda_{\mathrm{c}}(\boldsymbol{\Gamma})$ were obtained from determining the zeros of $q\left(H_{\lambda}(\boldsymbol{\Gamma})\right)$. At these lines, the Chern number changes by \pm 1 . Notice that the gap closings at $(0, \pi)$ and $(\pi, 0)$ coincide due to rotational symmetry. Secondly, there are transitions where the gap closes at four momenta away from the high symmetry points. In these cases, the Chern number changes by \pm 4 . Moreover, the transition lines corresponding to changes of the Chern number by \pm 4 always connect the thick lines corresponding to gap closings at high symmetry points (see Fig. 7).

The Hamiltonian obeys reflection symmetries with respect to the $x$ axis, the $y$ axis, and the two diagonals between these axes. As a consequence, $H$ is also fourfold rotationally symmetric. Therefore, the gap closings corresponding to changes of Chern number by \pm 4 always occur either (i) along the vertical and horizontal lines connecting $(0,0)$ to $(0, \pm \pi)$ and $( \pm \pi, 0)$ or (ii) along the diagonal lines connecting $(0,0)$ to $( \pm \pi, \pm \pi)$ points in the Brillouin zone. The lines denoted (a)-(c) and (d)-(f) in Fig. 7 correspond to these two different situations. To illustrate that this is indeed the case, we notice that the gap closings associated with topological phase transitions give rise to momentum space topological defects, so that we can visualize these topological defects (and gap closings) by plotting the Chern curvature of the impurity band in the vicinity of these phase transition lines as shown in Fig. 8.

We can understand also these gap closings occurring away from the high-symmetry points with the help of the zero dimensional $q\left(H_{\lambda}(\boldsymbol{k})\right)$. For this purpose we first notice that along these special lines in the Brillouin zone the zerodimensional Hamiltonian $H_{\lambda}(\boldsymbol{k})$ satisfies a chiral symmetry. The corresponding chiral symmetry operators can be written as $C=\tau_{y} M_{x}$ for $k_{y}=0, C=\tau_{x} M_{y}$ for $k_{x}=0, C=\left(\tau_{x}+\right.$ $\left.\tau_{y}\right) M_{x y} / \sqrt{2}$ for $k_{x}=k_{y}$, and $C=\left(\tau_{x}-\tau_{y}\right) M_{y x} / \sqrt{2}$ for $k_{x}=$ $-k_{y}$. Here $M_{x}, M_{y}, M_{x y}$, and $M_{y x}$ are matrices within the unit cell, which mirror with respect to the $x$ axis, $y$ axis, $y=x$ line, and $y=-x$ line, respectively. All these lines are defined in such a way that they run through the impurity site. Therefore, as explained in Sec. II A, we can use the chiral symmetry to write the Hamiltonian in the block off-diagonal form

$$
H_{\lambda}(\boldsymbol{k})=\left(\begin{array}{cc}
\mathbf{0} & D_{\lambda}(\boldsymbol{k}) \\
D_{\lambda}^{\dagger}(\boldsymbol{k}) & \mathbf{0}
\end{array}\right)
$$

and define a complex polynomial $q\left(H_{\lambda}(\boldsymbol{k})\right)=\operatorname{det} D_{\lambda}(\boldsymbol{k})$. It is easy to see that $q\left(H_{\lambda}(\boldsymbol{k})\right)$ is always linear in $\lambda$ but a nonlinear function of $\mu$. Therefore, there can exist some ranges of $\mu$, where a situation depicted in Fig. 3 is realized. In Fig. 9, we plot $q\left(H_{\lambda}(\boldsymbol{k})\right)$ as a function of $\lambda$ for $k_{x} a=k_{y} a=\pi / 2$ and $\mu \in[0.5 t, 1.5 t]$. The lines corresponding to $\mu=0.5 t$ and $\mu=1.5 t$ are on different sides of the origin, and by tuning $\mu \in[0.5 t, 1.5 t]$ one smoothly deforms the curve so that there exists a particular point $\mu=\mu_{c}=1.13 t$ and $\lambda=\lambda_{c}=3.47 t$ where $q\left(H_{\lambda}(\boldsymbol{k})\right)=0$. These values correspond to one point in the topological phase transition line denoted (d)-(f) in Fig. 7. The whole phase transition line is obtained by repeating this analysis for all $k_{x}=k_{y}=k$, where $k a \in[0, \pi]$. Because $q\left(H_{\lambda}(\boldsymbol{k})\right)$ is a nonlinear function of $\mu$ there can exist several

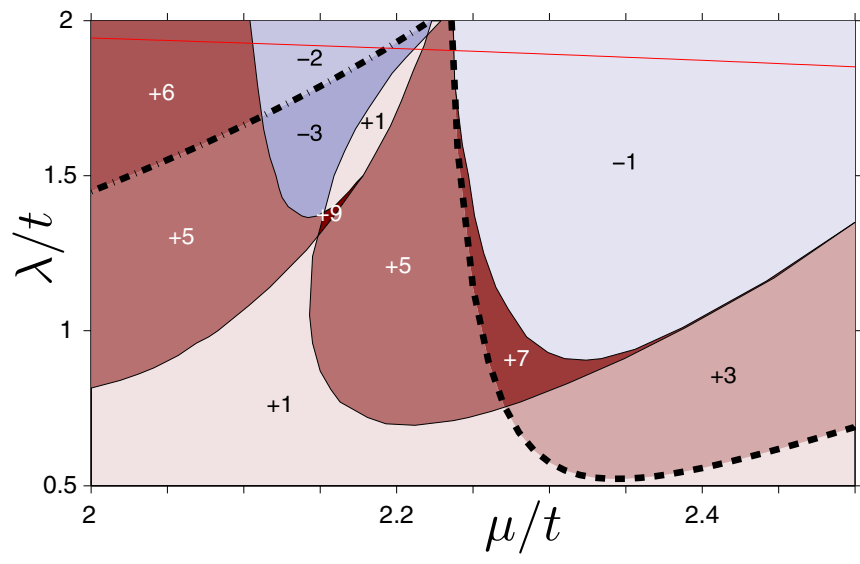

FIG. 10. Same as Fig. 7 but with parameters $\Delta=0.05 t$ and $a=5$.

ranges of $\mu$ where similar situations are realized. However, all the phase transition lines where the Chern number changes by \pm 4 (connecting the thick lines corresponding to gap closings at high symmetry points in Fig. 7) can be explained with a similar analysis of the parameter values where $q\left(H_{\lambda}(\boldsymbol{k})\right)=0$.

Finally, we find that even larger Chern numbers can be obtained by increasing the superconducting coherence length $\xi$ (by decreasing $\Delta$ ) and the lattice constant $a$; see Fig. 10. This is in agreement with Ref. [16]. There, it was argued that Chern numbers on the order of $\xi / a$ can be obtained in impurity lattices where $a$ is much larger than the lattice constant. In Fig. 10, there are also phase transition lines where the Chern number changes by 8 . These phase transition lines always connect the phase transition lines corresponding to gap closings at the special lines $k_{y}=0, k_{x}=0, k_{x}=k_{y}$, and $k_{x}=$ $-k_{y}$. The positions of the corresponding eight gap closings in the $\boldsymbol{k}$ space obey the rotational and reflection symmetries of the system. Therefore, the positions of the energy gap closings in the momentum space corresponding to the topological

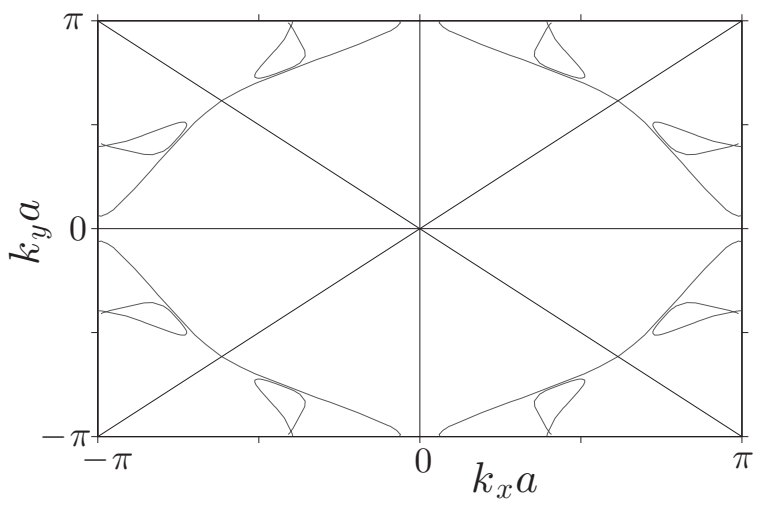

FIG. 11. The positions of the energy gap closings in the momentum space corresponding to the topological phase transition lines shown in Fig. 10 form a structure which resembles a spiderweb. The gap closings occurring along the lines $k_{y}=0, k_{x}=0, k_{x}=k_{y}$, and $k_{x}=-k_{y}$ form a support structure, and gap closing lines giving rise to changes of Chern number by 8 form curves which begin and end at these support lines. 
phase transition lines form a structure, which resembles a spiderweb (see Fig. 11). The gap closings occurring at high symmetry points and along the lines $k_{y}=0, k_{x}=0, k_{x}=k_{y}$, and $k_{x}=-k_{y}$ (possessing chiral symmetries) form the support structure of this spiderweb, and gap closing points giving rise to changes of Chern number by 8 form curves which begin and end at these support lines.

\section{Impurity-driven topological phase transitions in bilayer Chern insulators}

The $p_{x}+i p_{y}$ superconductor, similar to the ferromagnetic Shiba lattice model considered in Ref. [16], belongs to symmetry class D. However, it is clear that the particle-hole symmetry is not necessary for the appearance of large Chern numbers since small perturbations breaking this symmetry cannot affect the Chern number due to the presence of the energy gap. In this section, we study a closely related model, generically not supporting particle-hole symmetry, which we call bilayer Chern insulator. On one hand, this model allows us to establish how the breaking of the particle-hole symmetry affects the phase diagram. On the other hand, it allows us to generalize the interesting results concerning the impurity lattices also to topological insulators belonging to symmetry class A.

We assume that the Hamiltonian for a bilayer Chern insulator in the basis $c_{\boldsymbol{k}}^{\dagger}=\left(c_{1, \boldsymbol{k}}^{\dagger}, c_{2, \boldsymbol{k}}^{\dagger}\right)$ is

$$
H_{0}(\boldsymbol{k})=\left(\begin{array}{cc}
\xi_{1}(\boldsymbol{k}) & \mathcal{A}\left(\sin k_{x}+i \sin k_{y}\right) \\
\mathcal{A}\left(\sin k_{x}-i \sin k_{y}\right) & \xi_{2}(\boldsymbol{k})
\end{array}\right) .
$$

Here $c_{1(2), k}^{\dagger}$ are the electron creation operators for the different layers, $\xi_{1}=\mathcal{M}-2 t_{1}\left(\cos k_{x}+\cos k_{y}-2\right)-\mu$ and $\xi_{2}=-\mathcal{M}+2 t_{2}\left(\cos k_{x}+\cos k_{y}-2\right)-\mu$ are the dispersions for the energy bands in the different layers, $t_{1(2)}$ are the hopping amplitudes, $\mu$ is the chemical potential, $\mathcal{M}$ is the energy gap between bands $(\mathcal{M}<0$ means that the bands are inverted), and $\mathcal{A}$ describes the interlayer tunneling amplitude. We assume that the energy band in layer 1 is electronlike with positive effective mass, whereas the energy band in layer 2 is holelike with negative effective mass. The interlayer tunneling is assumed to be odd in momentum, which is naturally the case if the electronlike band is made out of $s$ orbitals and the holelike band from $p$ orbitals. Thus, our model describes a single spin block of the BHZ model [48], which may in principle be realized by coupling a quantum spin Hall insulator material to a ferromagnetic insulator to remove one of the spin blocks [49]. Furthermore, some of the quantum spin Hall insulator materials, such as the InAs/GaSb quantum wells [50], naturally have the bilayer structure, which will be important in the following. Namely, we will consider impurity lattices, where an impurity on lattice site $\boldsymbol{r}$ acts asymmetrically on the two layers $\lambda H_{1}=\lambda\left(c_{1 r}^{\dagger} c_{1 r}+u c_{2 r}^{\dagger} c_{2 r}\right)$. Here, $u$ describes the asymmetry of the impurity potential, and it can be controlled, for example, by placing different impurity atoms in the two layers at position $\boldsymbol{r}$. In the following we consider only the case $u<0$. This gives rise to two impurity bands originating from the two different layers, and the band inversions occurring between these impurity bands can cause topological phase

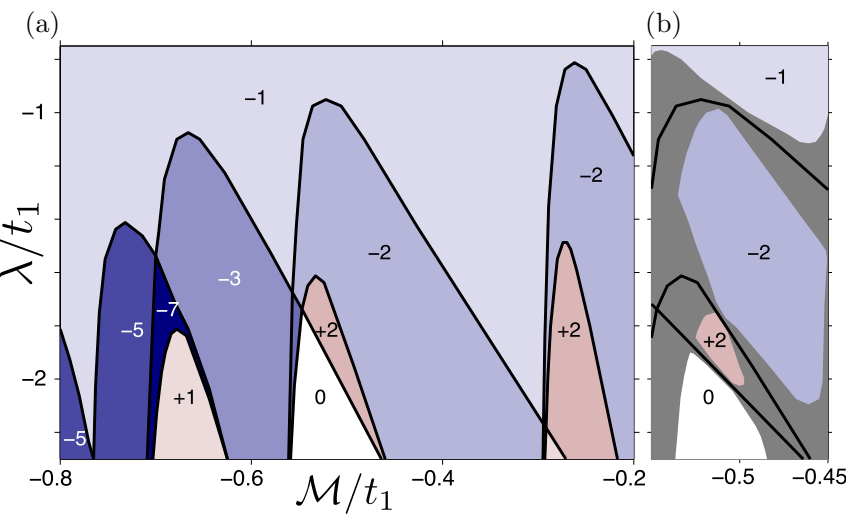

FIG. 12. Phase diagram of the bilayer Chern insulator in the presence of an impurity lattice as a function of $\lambda$ and $\mathcal{M}$ for $t_{2}=0.5 t_{1}$, $\mathcal{A}=0.05 t_{1}, a=7$, and $u=-1 / 2$. (a) For all values of $\mathcal{M}$ and $\lambda$ the chemical potential $\mu$ is tuned to be between the two impurity bands originating from the different layers. (b) The chemical potential is fixed to be $\mu=0.17 t_{1}$. The gray areas of the parameter space are gapless.

transitions. Similarly as earlier we arrange the impurities on a square lattice with lattice period $a$.

The phase diagram of the bilayer Chern insulator in the presence of an impurity lattice as a function of $\lambda$ and $\mathcal{M}$ is shown in Fig. 12 for $t_{2}=0.5 t_{1}, \mathcal{A}=0.05 t_{1}, a=7$, and $u=-1 / 2$. As can be seen from Fig. 12(a), despite the absence of particle-hole symmetry a rich variety of topologically distinct phases can be realized also in this model similarly as in the superconducting model analyzed in Sec. IV B. In Fig. 12(a), we have tuned the chemical potential to be between the impurity bands for all values of $\mathcal{M}$ and $\lambda$ in order to show the overall structure of the phase diagram for a large range of $\mathcal{M}$ and $\lambda$ values. However, there are also important differences to the $p_{x}+i p_{y}$ superconductor which become apparent when the chemical potential remains fixed as in Fig. 12(b). Namely, in the absence of particle-hole symmetry,

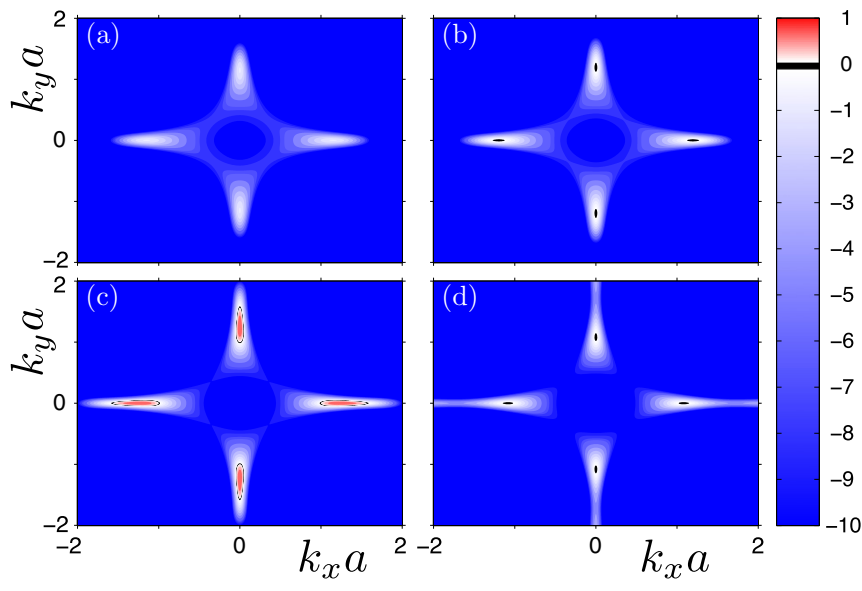

FIG. 13. $q\left(H_{\lambda}(\boldsymbol{k})\right)=\operatorname{det} H_{\lambda}(\boldsymbol{k}) \quad$ for $\quad \mathcal{M}=-0.5 t_{1} \quad$ and $\quad$ (a) $\lambda=-1.8 t$ (inside $C=-2$ phase), (b) $\lambda=-1.823 t$ (transition between $C=-2$ phase and gapless phase), (c) $\lambda=-1.9 t$ (inside gapless phase), (d) $\lambda=-1.96 t$ (transition between gapless phase and $C=2$ phase). The other parameters are the same as in Fig. 12(b). 
the topologically distinct phases generically are separated in parameter space by gapless phases. We can understand this generic feature with the help of $q\left(H_{\lambda}(\boldsymbol{k})\right)$ : In symmetry class A, there are no symmetries present and therefore the zero dimensional invariant is $q\left(H_{\lambda}(\boldsymbol{k})\right)=\operatorname{det} H_{\lambda}(\boldsymbol{k})$. Because $q\left(H_{\lambda}(\boldsymbol{k})\right)=\operatorname{det} H_{\lambda}(\boldsymbol{k})$ is a second order polynomial in $\lambda$, we generically expect that the topological phase transitions occur as illustrated for the transition between the $C=+2$ and $C=-2$ phases in Fig. 13. Inside the fully gapped topological phases, $q\left(H_{\lambda}(\boldsymbol{k})\right)$ has the same sign everywhere in momentum space as illustrated for the $C=+2$ phase in Fig. 13(a). By increasing $\lambda$ one can reach the transition point between the $C=+2$ phase and a gapless phase, and at this transition point $q\left(H_{\lambda}(\boldsymbol{k})\right)=0$ at a specific point $\boldsymbol{k}_{c}$ in the momentum space [Fig. 13(b)]. However, by further increasing $\lambda, q\left(H_{\lambda}(\boldsymbol{k})\right)$ generically changes sign in a region around $\boldsymbol{k}_{c}$ [Fig. 13(c)] indicating the appearance of zero-energy states (Fermi surface) at the boundary of this region, i.e., a gapless phase. When $\lambda$ is further increased, so that one approaches the $C=-2$ phase, the Fermi surface shrinks again and finally one reaches a specific value of $\lambda$, where $q\left(H_{\lambda}(\boldsymbol{k})\right)=0$ only at one point in the momentum space [Fig. 13(d)]. This defines the transition point between the gapless phase and the $C=-2$ phase. We thus find that generically the phase diagram for bilayer Chern insulator contains both gapless phases and fully gapped phases with a rich variety of Chern numbers. In addition to the gapless phases separating topologically distinct phases, there are also gapless phases describing semimetal phases with negative indirect gap. The relative amount of areas with gapless and fully gapped phases in the parameter space strongly depends on $u$.

\section{SUMMARY AND DISCUSSION}

In summary, we have identified the general conditions under which the eigenenergies of the Hamiltonian undergo a robust zero-energy crossing as a function of external parameters in different Altland-Zirnbauer symmetry classes. We defined $q(H)$ as a generalized root of $\operatorname{det} H$ and used it to predict or to rule out, respectively, robust zero-energy crossings in all symmetry classes. We complemented this result with a perturbation theory analysis, which allows for a derivation of the asymptotic low-energy behavior of the ensemble averaged density of states $\rho \sim E^{\alpha}$ for all symmetry classes. Finally, we have utilized $q(H(\boldsymbol{k}))$ to show that a lattice of impurities can drive a topologically trivial system into a nontrivial phase and revealed impurity bands carrying extremely large Chern numbers in different symmetry classes of two-dimensional topological insulators and superconductors. This analysis makes it transparent that $q(H(\boldsymbol{k}))$ can be used as a powerful tool in the analysis of topological phase transitions in higher dimensional systems.

The impurity bands carrying large Chern number $C$ are interesting on their own. In the bilayer Chern insulator, they will support a large number $(|C|)$ of topologically protected fermionic edge modes allowing dissipationless transport, and in topological superconductors there will be $|C|$ Majorana edge modes. However, we also want to point out that the bandwidth of the impurity bands can be quite small, and it may be tuned by varying the distance between impurities. Therefore, these impurity bands carrying large Chern numbers can potentially also support interesting correlated phases in the presence of interactions. In particular, the interactions may lead to spontaneous symmetry breaking, such as superconductivity, ferromagnetism, or exciton condensation, and the physical properties, such as the charge of topological defects and superfluid density, are expected to depend on $C$ [51-54]. Moreover, partially filled nearly flat bands with a large Chern number in the presence of interactions may lead to exotic strongly correlated states of matter with interesting topological properties [55-57].

\section{ACKNOWLEDGMENTS}

L.K. acknowledges valuable discussions with H.-G. Zirnstein. This work was supported by DFG Grant No. RO 2247/8-1, by the Academy of Finland through its Center of Excellence program, and by the European Research Council (Grant No. 240362-Heattronics).

\section{APPENDIX A: TOPOLOGICAL INVARIANTS IN THE PRESENCE OF AN ADDITIONAL SYMMETRY $U$ ANTICOMMUTING WITH $C$}

In this section, we derive the block-diagonal structure for $H$ satisfying a chiral symmetry in the presence of an additional symmetry $[H, U]=\{C, U\}=0$, with $U^{\dagger} U=1$. Symmetries of that kind have been considered before for class DIII in Refs. [28,40]. The complementary case where $U$ and $C$ commute was studied in Ref. [41].

The simultaneous eigenstates of $H$ and $U, H|E, u\rangle=$ $E|E, u\rangle$ and $U|E, u\rangle=u|E, u\rangle$ satisfy

$$
C|E, u\rangle=|-E,-u\rangle .
$$

Choosing the states $|E, u\rangle$ as a basis of the Hilbert space and sorting them appropriately, the operators $U, H$, and $C$ become block diagonal:

$$
\begin{aligned}
U & =\bigoplus_{i=1}^{n}\left(\begin{array}{cc}
u_{i} \mathbf{1}_{m_{i}} & \mathbf{0}_{m_{i}} \\
\mathbf{0}_{m_{i}} & -u_{i} \mathbf{1}_{m_{i}}
\end{array}\right), \\
H & =\bigoplus_{i=1}^{n}\left(\begin{array}{ll}
H_{+i} & \mathbf{0}_{m_{i}} \\
\mathbf{0}_{m_{i}} & H_{-i}
\end{array}\right), \\
C & =\bigoplus_{i=1}^{n}\left(\begin{array}{cc}
\mathbf{0}_{m_{i}} & C_{i} \\
C_{i}^{\dagger} & \mathbf{0}_{m_{i}}
\end{array}\right) .
\end{aligned}
$$

Here, $m_{i}$ denotes the degree of degeneracy of the eigenvalue $u_{i}$. The specific form of $C$ follows from Eq. (A1) and the property $C^{\dagger} C=C^{2}=1$. Each of the Hamiltonian's blocks $H_{ \pm i}$ lacks chiral symmetry, although the full Hamiltonian still has chiral symmetry which requires $H_{-i}=-C_{i}^{\dagger} H_{+i} C_{i}$. Therefore the number of SPEs per block is halved from the original value $v_{ \pm i}=v / 2$. Consequently, the blocks $H_{ \pm i}$ belong to a different symmetry class than $H$, namely to (i) A when $H$ belongs to AIII, (ii) A or AI when $H$ belongs to CI, (iii) A or AI when $H$ belongs to BDI, (iv) D or AII when $H$ belongs to DIII, and (v) $\mathrm{C}$ or AII when $H$ belongs to CII. So in classes AIII, CI, and DIII the symmetry $U$ prevents level repulsion and enables the definition of an invariant $\mathcal{Q}_{U}=\left(\mathcal{Q}_{H_{+1}}, \ldots, \mathcal{Q}_{H_{+n}}\right)$, which is the vector of invariants of independent blocks $H_{+i}$. This is 
not necessarily the case when $H$ is from CII, since in this case all blocks can belong to class $\mathrm{C}$.

\section{APPENDIX B: IMPOSSIBILITY OF DEFINING $q(H)$ IN CLASSES C AND CII}

In symmetry classes $\mathrm{C}$ and $\mathrm{CII}$, it is not possible to calculate the generalized second and fourth root of det $H$, respectively. To show this, it suffices to demonstrate the impossibility of defining a polynomial expression $q(H)$ in the matrix elements of a Hamiltonian from class $\mathrm{C}$, which would satisfy $|\operatorname{det} H|=$ $|q(H)|^{2}$. From this, the impossibility of defining $q(H)$ for arbitrary Hamiltonians in class CII follows, because a simple Hamiltonian in CII can, for example, be the direct sum $H_{\mathrm{CII}}^{0}=$ $H_{\mathrm{C}} \oplus H_{\mathrm{C}}^{\prime}$ of two Hamiltonians from class $\mathrm{C}$ which are related to each other by TR, $H_{\mathrm{C}}^{\prime}=T H_{\mathrm{C}} T^{-1}$ with $T^{2}=-1$.

The simplest Hamiltonian in class $\mathrm{C}$ reads $H_{\mathrm{C}}^{0}=-\boldsymbol{B} \cdot \boldsymbol{\sigma}$ [58]. Here, $\boldsymbol{B}=\left(\operatorname{Re} b_{\perp}, \operatorname{Im} b_{\perp}, b_{\|}\right)$and $\boldsymbol{\sigma}=\left(\sigma_{1}, \sigma_{2}, \sigma_{3}\right)$ is the vector of Pauli matrices. Considering

$$
\left|\operatorname{det} H_{\mathrm{C}}^{0}\right|=\left.\left|b_{\|}+i\right| b_{\perp}\right|^{2}
$$

it becomes manifest that any candidate for $q(H)$ in class $\mathrm{C}$ would involve the calculation of absolute values or square roots, like $\left|b_{\perp}\right|$ or $\sqrt{b_{\perp}^{*} b_{\perp}}$, respectively. Hence, there is no expression $q(H)$ in class $\mathrm{C}$ that meets the criterion of being a polynomial expression in the Hamiltonian's matrix elements.

\section{APPENDIX C: CALCULATIONS FOR THE KITAEV CHAIN}

This section contains details about the derivation of the results for the Kitaev chain with impurity lattice in Sec. IV A. We consider the translationally invariant Hamiltonian $H_{\lambda}=$ $H_{0}+\lambda H_{1}^{\prime}$ with $H_{0}$ given by Eq. (14) and $\lambda H_{1}^{\prime}=\lambda \sum_{i} c_{a i}^{\dagger} c_{a i}$. For the Kitaev chain, bringing $H_{\lambda}$ into the block off-diagonal form of Eq. (4b) corresponds to transforming to the Majorana basis $\left(\gamma_{A, 1}, \ldots, \gamma_{A, N}, \gamma_{B, 1}, \ldots, \gamma_{B, N}\right)$ where $\gamma_{A, j}=\frac{1}{\sqrt{2}}\left(c_{j}+\right.$ $\left.c_{j}^{\dagger}\right)$ and $\gamma_{B, j}=\frac{i}{\sqrt{2}}\left(c_{j}-c_{j}^{\dagger}\right)$ [31]. In momentum space, the offdiagonal block $D_{\lambda}(\Gamma)$ at the high symmetry points $\Gamma=0, \pi$ reads

$$
D_{\lambda}(\Gamma)=-i\left(\begin{array}{cccc}
-\mu+\lambda & -2 t & & \\
0 & -\mu & \ddots & \\
& \ddots & \ddots & -2 t \\
-2 t e^{i \Gamma} & & 0 & -\mu
\end{array}\right)
$$

with matrix dimension $a$. It is then straightforward to determine the critical impurity strengths [Eq. (15)] from the condition det $D_{\lambda_{\mathrm{c}}}=0$. Moreover, the corresponding normalized eigenvector $\psi$ satisfying $D_{\lambda_{\mathrm{c}}} \psi=0$ is

$$
\psi=\sqrt{\frac{\beta^{2}-1}{\beta^{2 a}-1}}\left(e^{-i \Gamma}, \beta^{a-1}, \beta^{a-2}, \ldots, \beta\right)^{T},
$$

where $\beta=-2 t / \mu$. From these eigenvectors, one obtains the decay length $\xi^{\infty}=1 / \ln |\mu / 2 t|$.
[1] M. Z. Hasan and C. L. Kane, Rev. Mod. Phys. 82, 3045 (2010).

[2] X.-L. Qi and S.-C. Zhang, Rev. Mod. Phys. 83, 1057 (2011).

[3] A. Y. Kitaev, Phys.-Usp. 44, 131 (2001).

[4] C. L. Kane and E. J. Mele, Phys. Rev. Lett. 95, 146802 (2005).

[5] L. Fu, C. L. Kane, and E. J. Mele, Phys. Rev. Lett. 98, 106803 (2007).

[6] J. E. Moore and L. Balents, Phys. Rev. B 75, 121306(R) (2007).

[7] R. Roy, Phys. Rev. B 79, 195322 (2009).

[8] A. P. Schnyder, S. Ryu, A. Furusaki, and A. W. W. Ludwig, Phys. Rev. B 78, 195125 (2008).

[9] A. Kitaev, AIP Conf. Proc. 1134, 22 (2009).

[10] T. Hyart, A. R. Wright, G. Khaliullin, and B. Rosenow, Phys. Rev. B 85, 140510 (2012).

[11] S. Okamoto, Phys. Rev. B 87, 064508 (2013).

[12] D. D. Scherer, M. M. Scherer, G. Khaliullin, C. Honerkamp, and B. Rosenow, Phys. Rev. B 90, 045135 (2014).

[13] Y.-Z. You, I. Kimchi, and A. Vishwanath, Phys. Rev. B 86, 085145 (2012).

[14] A. M. Black-Schaffer, W. Wu, and K. Le Hur, Phys. Rev. B 90, 054521 (2014).

[15] L. Du, I. Knez, G. Sullivan, and R.-R. Du, Phys. Rev. Lett. 114, 096802 (2015).

[16] J. Röntynen and T. Ojanen, Phys. Rev. Lett. 114, 236803 (2015).

[17] J. Li, R.-L. Chu, J. K. Jain, and S.-Q. Shen, Phys. Rev. Lett. 102, 136806 (2009).

[18] C. W. Groth, M. Wimmer, A. R. Akhmerov, J. Tworzydło, and C. W. J. Beenakker, Phys. Rev. Lett. 103, 196805 (2009).
[19] İ. Adagideli, M. Wimmer, and A. Teker, Phys. Rev. B 89, 144506 (2014).

[20] W. Qin, D. Xiao, K. Chang, S.-Q. Shen, and Z. Zhang, arXiv: 1509.01666.

[21] J. D. Sau and E. Demler, Phys. Rev. B 88, 205402 (2013).

[22] R.-J. Slager, L. Rademaker, J. Zaanen, and L. Balents, Phys. Rev. B 92, 085126 (2015).

[23] G. E. Volovik, The Universe in a Helium Droplet (Clarendon Press, Oxford, 2003).

[24] A. Altland and M. R. Zirnbauer, Phys. Rev. B 55, 1142 (1997).

[25] M. R. Zirnbauer, J. Math. Phys. 37, 4986 (1996).

[26] C. W. J. Beenakker, J. M. Edge, J. P. Dahlhaus, D. I. Pikulin, S. Mi, and M. Wimmer, Phys. Rev. Lett. 111, 037001 (2013).

[27] I. C. Fulga, F. Hassler, and A. R. Akhmerov, Phys. Rev. B 85, 165409 (2012).

[28] L. Kimme, T. Hyart, and B. Rosenow, Phys. Rev. B 91, 220501(R) (2015).

[29] M. Marinescu and A. Dalgarno, Z. Phys. D: At., Mol. Clusters 36, 239 (1996).

[30] F. Haake, Quantum Signatures of Chaos, 3rd ed. (Springer, Berlin, Heidelberg, 2010), Chap. 3; M. V. Berry, in Les Houches, Session XXXVI, 1981m Chaotic Behavior of Deterministic Systems (North-Holland, Amsterdam, 1983).

[31] C. W. J. Beenakker, Rev. Mod. Phys. 87, 1037 (2015).

[32] D. A. Ivanov, J. Math. Phys. 43, 126 (2002).

[33] W. P. Su, J. R. Schrieffer, and A. J. Heeger, Phys. Rev. Lett. 42, 1698 (1979).

[34] L. Fu, Phys. Rev. Lett. 106, 106802 (2011). 
[35] C. Fang, M. J. Gilbert, and B. A. Bernevig, Phys. Rev. B 86, 115112 (2012).

[36] R.-J. Slager, A. Mesaros, V. Juricic, and J. Zaanen, Nat. Phys. 9, 98 (2013).

[37] Y. Ueno, A. Yamakage, Y. Tanaka, and M. Sato, Phys. Rev. Lett. 111, 087002 (2013).

[38] F. Zhang, C. L. Kane, and E. J. Mele, Phys. Rev. Lett. 111, 056403 (2013).

[39] C.-K. Chiu, H. Yao, and S. Ryu, Phys. Rev. B 88, 075142 (2013).

[40] K. Wölms, A. Stern, and K. Flensberg, Phys. Rev. Lett. 113, 246401 (2014).

[41] M. Koshino, T. Morimoto, and M. Sato, Phys. Rev. B 90, 115207 (2014).

[42] P. Ghosh, J. D. Sau, S. Tewari, and S. Das Sarma, Phys. Rev. B 82, 184525 (2010).

[43] In the case of $\operatorname{tr} C=n \in \mathbb{Z} \backslash\{0\}$, the present discussion is not of interest anyway, because there will be $|n|$ zero-energy states protected by the chiral symmetry, so that $\operatorname{det} H \equiv 0$.

[44] A. V. Balatsky, I. Vekhter, and J.-X. Zhu, Rev. Mod. Phys. 78, 373 (2006).

[45] Except for the definitions of the Hamiltonians $H_{0}$ in Eqs. (6) and (17), which do not contain an impurity lattice, all discussion in Sec. IV refers to the reduced Brillouin zone in the presence of an impurity lattice.

[46] S. Tewari and J. D. Sau, Phys. Rev. Lett. 109, 150408 (2012).
[47] The Chern numbers in Figs. 7, 10, and 12 were obtained by connecting points in the gapped phases with $\lambda \neq 0$ to points at the lines $\lambda=0$ and calculating the change $\Delta C_{\text {imp }}$ of the impurity band's Chern number at each gap closing line.

[48] B. A. Bernevig, T. L. Hughes, and S.-C. Zhang, Science 314, 1757 (2006).

[49] Without the ferromagnetic insulator to break time-reversal symmetry, the physics discussed here can still be realized, but then the two spin blocks will give rise to opposite Chern numbers.

[50] C. Liu, T. L. Hughes, X.-L. Qi, K. Wang, and S.-C. Zhang, Phys. Rev. Lett. 100, 236601 (2008).

[51] S. L. Sondhi, A. Karlhede, S. A. Kivelson, and E. H. Rezayi, Phys. Rev. B 47, 16419 (1993).

[52] K. Moon, H. Mori, K. Yang, S. M. Girvin, A. H. MacDonald, L. Zheng, D. Yoshioka, and S.-C. Zhang, Phys. Rev. B 51, 5138 (1995).

[53] D. I. Pikulin, P. G. Silvestrov, and T. Hyart, arXiv:1504.05154.

[54] S. Peotta and P. Törmä, Nat. Commun. 6, 8944 (2015).

[55] M. Barkeshli and X.-L. Qi, Phys. Rev. X 2, 031013 (2012).

[56] Z. Liu, E. J. Bergholtz, H. Fan, and A. M. Läuchli, Phys. Rev. Lett. 109, 186805 (2012).

[57] A. Sterdyniak, C. Repellin, B. A. Bernevig, and N. Regnault, Phys. Rev. B 87, 205137 (2013).

[58] The constituting $\mathrm{PH}$ symmetry of class $\mathrm{C}$ follows from $\left\{H_{\mathrm{C}}^{0}, i \sigma_{2} K\right\}=0$ and $\left(i \sigma_{2} K\right)^{2}=-1$. 